\section{Flow diverter stents in the treatment of recanalized intracranial aneurysms}

\author{
Erol Akgul ${ }^{1}$ (D), Hasan Bilen Onan ${ }^{2}$, Irem Islek ${ }^{3}$, Mehmet Tonge ${ }^{4}$, \\ Yavuz Durmus ${ }^{5}$, Mehmet Barburoglu ${ }^{6}$, Aynur Azizova ${ }^{7}$, Cengiz Erol ${ }^{3}$, \\ Bahattin Hakyemez $^{8}$, Serra Sencer ${ }^{6}$, Kubilay Aydin ${ }^{6,9}$ and Anil Arat ${ }^{7}$
}

Interventional Neuroradiology

1-9

(C) The Author(s) 2021

Article reuse guidelines:

sagepub.com/journals-permissions

DOI: $10.1177 / 1591019921990507$

journals.sagepub.com/home/ine

\section{@SAGE}

\begin{abstract}
Background: We assessed the safety and efficacy of flow diverter stents (FDSs) in the treatment of recanalized or residual intracranial aneurysms treated endovascularly.

Materials \& Methods: Patients whose recanalized or residual aneurysms were treated with FDSs in five tertiary hospitals were reviewed retrospectively. The patients' demographic data, aneurysm characteristics, types of previous treatment, and clinical complications, or serious adverse events associated with FDSs, as well as the results of neurological and angiographic follow-up assessments, were recorded.

Results: Eighty-six patients (37 males) with 87 aneurysms were included in this study. Eighty (91.9\%) aneurysms were in the anterior and seven (8.1\%) in the posterior circulation. The initial treatment methods were the primary coiling or balloon remodeling technique in $69(79.3 \%)$ and stent-assisted coiling in $18(20.7 \%)$ aneurysms. The endovascular procedure was successful in all patients. Complications occurred in four patients, for a total complication rate of $4.6 \%$. A technical complication developed in one patient $(1.2 \%)$. An in-stent thrombosis treated with tirofiban was seen in two cases. Late in-stent stenosis exceeding $50 \%$ was treated with balloon angioplasty in one patient. The mean length of follow-up was 21.0 months. The first angiographic follow-up (3-6 months) revealed the complete occlusion of 74 aneurysms (85.1\%). While 76 aneurysms (87.4\%) were occluded at the last angiographic follow-up (mean: 26.0 months), 11 aneurysms (12.6\%) were still filling. Morbimortality was zero.

Conclusion: The drawback of endovascular treatment is aneurysmal remnants or recurrences, which is safely and durably amenable to flow diversion.
\end{abstract}

\title{
Keywords
}

Recanalization, cerebral aneurysm, residual aneurysm, endovascular treatment, flow diverter

Received 1 October 2020; revised 8 December 2020; accepted 2 January 2021

\section{Introduction}

Flow diversion is a recently described endovascular method for the treatment of intracranial aneurysms. The safety and efficacy of flow diversion treatment have been proven for the treatment of large and wide-necked carotid siphon aneurysms. ${ }^{1-7}$ Following the promising results of the studies that reported the clinical and angiographic outcomes of flow diversion treatment, flow diversion treatment was recently found to help treat very small aneurysms; ruptured aneurysms such as blister-like examples; and even bifurcation aneurysms located distal to the Circle of Willis. $^{8-12}$

The recurrence of the aneurysms treated with only coiling or stent-assisted coiling, or the remnant of ruptured aneurysms that have been coiled is not infrequent, and the results of retreatment with
${ }^{1}$ Interventional Neuroradiology Section, Radiology Department, International School of Medicine, Istanbul Medipol University, Istanbul, Turkey

${ }^{2}$ Radiology Department, School of Medicine, Cukurova University, Adana, Turkey

${ }^{3}$ Radiology Department, School of Medicine, Istanbul Medipol University, Istanbul, Turkey

${ }^{4}$ Neurosurgery Department, School of Medicine, Istanbul Medipol University, Istanbul, Turkey

${ }^{5}$ Radiology Department, Bursa Yuksek Ihtisas Education and Research Hospital, Bursa, Turkey

${ }^{6}$ Department of Neuroradiology, Istanbul Faculty of Medicine, Istanbul University, Istanbul, Turkey

${ }^{7}$ Radiology Department, School of Medicine, Hacettepe University, Ankara, Turkey

${ }^{8}$ Radiology Department, School of Medicine, Uludag University, Bursa, Turkey

${ }^{9}$ Department of Interventional Neuroradiology, Faculty of Medicine, Koc University, Istanbul, Turkey

Corresponding author:

Erol Akgul, Istanbul Medipol Universitesi, Tıp Fakultesi, Radyoloji ABD, Bagcilar, Istanbul, Turkey.

Email: akgulerol@gmail.com 
conventional techniques are not satisfactory. The safety and efficacy of flow diversion in the treatment of almost any kind of aneurysm make it a salvageable treatment method for aneurysms. ${ }^{13}$ However, data in the literature about the endovascular treatment of recanalized and residual aneurysms with flow diversion are limited. ${ }^{13-16}$ In this retrospective, single-arm, and multicenter study, we assessed the safety and efficacy of the flow diverter stents (FDSs) for the treatment of recanalized or residual intracranial aneurysms that had been treated by endovascular techniques other than flow diverters.

\section{Materials and methods}

\section{Patient selection}

After obtaining institutional ethics committee approval, we retrospectively reviewed the databases of five centers to identify all patients with a recanalized or residual intracranial aneurysm who were treated between 2008 and 2019 with implantation of FDSs. The centers provided unidentified data only for patients who underwent a flow diversion treatment for the recanalized or residual aneurysms that had been previously treated with other endovascular techniques. In general flow diversion was preferred to treat recanalized sidewall aneurysms. However, some recanalized aneurysms treated by other techniques, including previously stented aneurysms in which the wall apposition of the stent was questionable, as well as the majority of the bifurcation aneurysms treated by crossing stents and finally those bifurcation aneurysms which were deemed to be amenable to durable occlusion with further coiling (with or without stent assistance) were not treated by flow diversion and hence were not included in the analysis. Patients with recanalized aneurysms after previous endosaccular treatments who are unlikely to be compliant with dual antiplatelet therapy were also not offered treatment with FDSs. The decision to perform any endovascular treatment including flow diversion was made in cases of a continuous increase of recanalized or residual part of the aneurysms treated with coiling or stent-assisted coiling. The patients' demographics, aneurysm characteristics, treatment histories, clinical complications, or serious adverse events associated with flow diversion, and the results of neurological and angiographic follow-up assessment were recorded. Aneurysm occlusion was evaluated as "complete" or "not".

\section{Endovascular procedure}

Although there were some differences in the endovascular procedures and follow-up protocols, the basic approach used by the participating centers was similar. All procedures were treated as elective, and patients were premedicated before the interventions.
Depending on the preference of each center, daily $100-300 \mathrm{mg}$ of aspirin, and either $75 \mathrm{mg}$ of clopidogrel or $10 \mathrm{mg}$ of prasugrel, was started at least 5 days before the endovascular procedure. The response to the clopidogrel/prasugrel was verified before the procedure with a point-of-care assay. Patients with an inadequate response to clopidogrel were switched to prasugrel with a starting daily dose of $10 \mathrm{mg}$. All endovascular procedures were performed under general anesthesia using a femoral approach. Systemic anticoagulation was initiated immediately after the insertion of a femoral introducer sheath with a bolus dose of $5000 \mathrm{IU}$ of IV heparin or 70-100 IU/ $\mathrm{kg}$ heparin based on operator preference. The bolus dose was followed by a slow heparin infusion or a 1000 IU IV bolus dose per hour to maintain an activated clotting time that was approximately 2-fold greater than the baseline value. Access to the target artery (internal carotid or vertebral artery) was achieved with a neurovascular distal access catheter after a long sheath insertion. The distal tip of a $0.021-$ 0.027 -inch microcatheter was positioned at the parent artery distal to the aneurysm neck. Then, an FDS [Silk, Balt, Montmorency, France; Derivo, Acandis, Pforzheim, Germany; Surpass, Stryker Neuroendovascular, Kalamazoo, MI, USA; FRED, Microvention Terumo, Tustin, CA, USA, Pipeline Embolization Device (PED), Medtronic Covidien AG, Paris, France] was deployed to cover the neck of the aneurysm. Expansion and wall apposition of the deployed stent was assessed under fluoroscopy and cone-beam CT if needed. In cases of incomplete stent expansion, we performed in-stent balloon angioplasty to achieve a full wall apposition; in one center, stenting with a non-FDS was used to treat malapposition. Post-procedural dual antiplatelet therapy was continued for 6 months and was switched to daily aspirin thereafter.

\section{Follow-up}

At the end of the procedure, a cone-beam CT was performed to identify any procedural hemorrhagic complications before waking up the patient. The first follow-up digital subtraction angiography (DSA) was performed at 3-6 months. The second follow-up angiogram, either invasive or noninvasive, was performed at 9-12 months. Patients' neurological status was evaluated during discharge and at the angiographic follow-up using the mRS scale.

\section{Statistical analysis}

All data are presented as means and ranges for continuous variables and as frequencies for categorical variables. A Chi-square test was performed to test the significance of the differences between subgroups. The statistical analysis was performed using SPSS statistics (IBM, Armonk, New York). 


\section{Results}

Eighty-six patients (37 males) with 87 aneurysms were included in this study. The mean age of the patients was 50.0 years (range, 11-78 years). Eighty (91.9\%) aneurysms were in the anterior and $7(8.1 \%)$ were in the posterior circulation. Detailed locations of the aneurysms and how they were previously treated are shown in Table 1, in addition to demographic features. The mean time between the initial treatment and FDS insertion was 21 months. The initial treatment methods were the primary coiling or balloon remodeling technique in $69(79.3 \%)$ aneurysms and stent-assisted coiling in $18(20.7 \%)$ aneurysms.

The endovascular procedure was successful in all patients. In four patients $(4.6 \%)$, peri- and postprocedural complications were encountered. A technical complication developed in one patient $(1.2 \%)$. In this patient, who had a posterior communicating artery ruptured aneurysm previously treated with only coiling, a Leo stent (Balt) was deployed telescopically inside the Silk stent to correct malapposition. The technical complications remained asymptomatic,

Table 1. Patient data and aneurysm characteristics.

\begin{tabular}{lc}
\hline & $N(\%)$ \\
\hline Number of patients & \\
Age, years & \\
Mean & \\
Sex & \\
Male & \\
Female & $37(43.0))$ \\
Recanalized aneurysms, total & $49(57.0)$ \\
Ruptured & $87(100.0)$ \\
Unruptured & $65(74.7)$ \\
Aneurysm initial size (mean) & $22(25.3)$ \\
Treated with coils & $3-40 \mathrm{~mm}(10.3)$ \\
Treated with stent and coils & $69(79.3)$ \\
$\quad$ With braided & $18(20.7)$ \\
$\quad$ With laser-cut & $9(50.0)$ \\
Aneurysm location & $9(50.0)$ \\
Anterior circulation & \\
ICA cavernous & $80(91.9)$ \\
ICA ophthalmic & $2(2.3)$ \\
PComA & $41(47.1)$ \\
AChorA & $17(19.5)$ \\
ICA terminal & $4(4.6)$ \\
MCA & $3(3.4)$ \\
ACA & $8(9.2)$ \\
ACA, distal & $2(2.3)$ \\
AComA & $2(2.3)$ \\
Posterior circulation & $1(1.1)$ \\
PICA & $7(8.1)$ \\
Vertebrobasilar & $2(2.3)$ \\
Basilar trunk & $1(1.1)$ \\
Basilar tip & $2(2.3)$ \\
\hline
\end{tabular}

$\mathrm{N}$ : number; ICA: internal carotid artery; PComA: posterior communicating artery; AChorA: anterior choroidal artery; MCA: middle cerebral artery; ACA: anterior cerebral artery; AComA: anterior communicating artery; PICA: posterior inferior cerebellar artery. and the mRS scores of these patients remained zero at discharge. No hemorrhagic complications were detected on CT performed immediately following the procedure. We observed a periprocedural thrombotic complication in two patients (2.3\%). Intraprocedural control DSA images revealed the development of an in-stent thrombus. Intra-arterial infusion of tirofiban through the microcatheter achieved complete resolution of the thrombus in both cases. These patients did not develop any neurological symptoms, and their mRS scores were zero at discharge. We observed a delayed complication in one patient $(1.2 \%)$, who had a recanalized ICA ophthalmic segment aneurysm treated with a Silk stent, 1 year later. The follow-up DSA examination of this patient revealed in-stent stenosis $(>50 \%)$, which responded well to balloon angioplasty. The morbimortality was zero. Table 2 shows the procedural and follow-up data, as well as complications. At least one angiographic follow-up was performed in all patients. The mean length of follow-up was 26.0 months (range, 3-92 months). The first angiographic follow-up (3-6 months) revealed the complete occlusion of 74 aneurysms $(85.1 \%)$. While 76 aneurysms

Table 2. Procedural and follow-up data, and complications.

\begin{tabular}{lr}
\hline & N (mean or \%) \\
\hline FDSs, total used & $87(100.0)$ \\
Silk & $60(69.0)$ \\
Derivo & $9(10.3)$ \\
Surpass & $7(8.1)$ \\
FRED & $6(6.9)$ \\
Pipeline & $5(5.7)$ \\
Number of FD per aneurysm & 1.0 \\
Time between initial treatment and FDS (month) & $2-96(21.0)$ \\
Follow-up duration, angiographic (month) & $3-92(26.0)$ \\
Closure rate, at 3-6th month & \\
Complete & $74(85.1)$ \\
Incomplete & $13(14.9)$ \\
Closure rate, overall & \\
Complete & $76(87.4)$ \\
$\quad$ Aneurysm initial size & $3-40(10.0)$ \\
Incomplete & $11(12.6)$ \\
$\quad$ Aneurysm initial size & $4-35$ mm (14.7) \\
Closure rate in coiled aneurysms & \\
Complete & $62(89.9)$ \\
Incomplete & $7(10.1)$ \\
Closure rate in stented aneurysms & \\
Complete & $14(77.8)$ \\
Incomplete & $4(22.2)$ \\
$\quad$ With braided stent & $1(25.0)$ \\
With laser-cut stent & $3(75.0)$ \\
Complications, total (per patient) & $4(4.6)$ \\
Technical & $1(1.2)$ \\
Stent thrombosis & $2(2.3)$ \\
Stent stenosis & $1(1.2)$ \\
Morbidity & 0 \\
Mortality & 0 \\
\hline
\end{tabular}

$\mathrm{N}$ : number; FDS: flow diverter stent. 
$(87.4 \%)$ were occluded at the last angiographic follow-up (3-92 months, mean: 26.0), 11 aneurysms $(12.6 \%)$ were still filling. The mean follow-up time of five of 11 aneurysms with the persistent filling was 16 months (range, 3-33 months). The locations of the aneurysms that still had not occluded completely were the internal carotid artery ophthalmic segment in four, the posterior communicating artery in two, the middle cerebral artery in two, the anterior cerebral artery in one, and the basilary tip in two patients. Table 2 also shows the closure rates for each subgroup of patients when the patients were grouped with regard to the initial treatment type.

Chi-square tests performed for closure rates of subgroups showed no significant differences among them (p: 0.231).

Figures 1 and 2 show representative cases.

\section{Discussion}

Unlike endosaccular treatments, parent artery endoluminal reconstruction with FDSs for the treatment

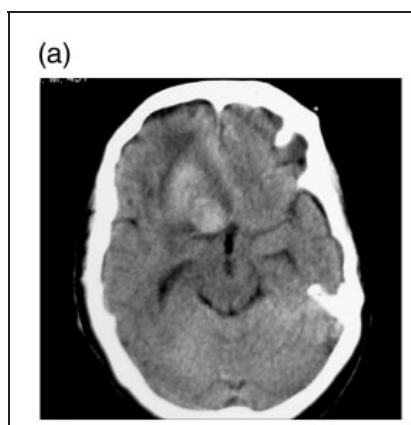

(e)

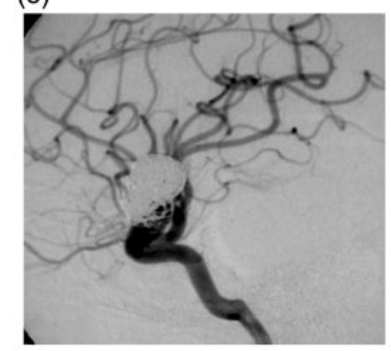

(i)

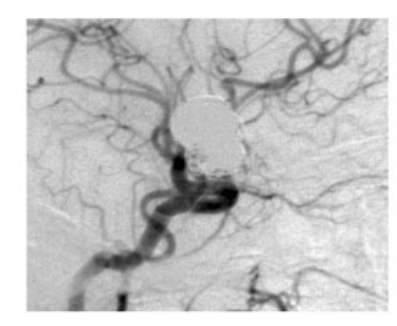

(b)

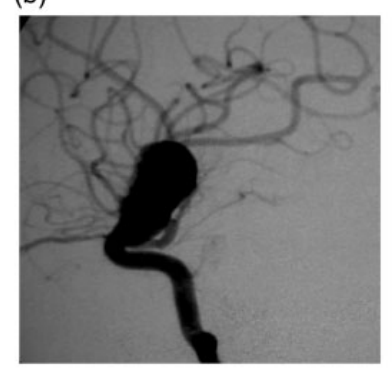

(f)

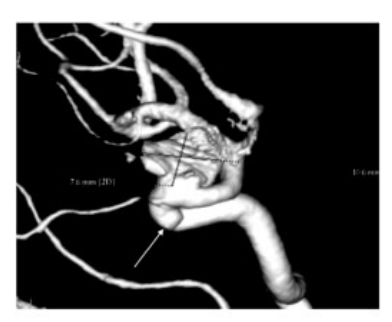

(j)

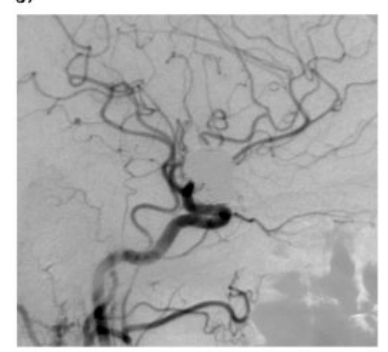

(c)

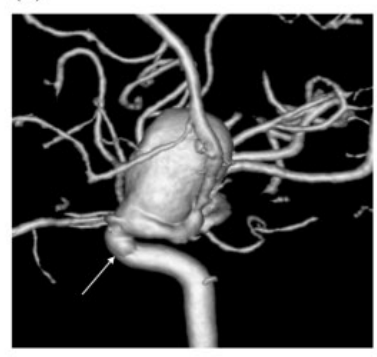

(g)

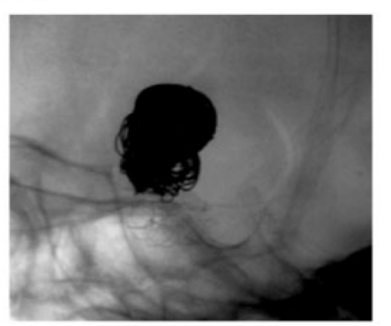

(k)

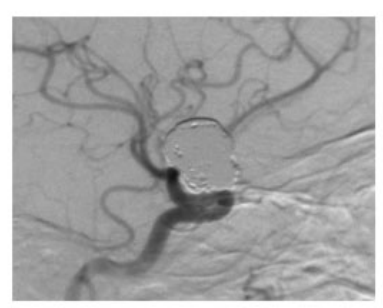

(d)

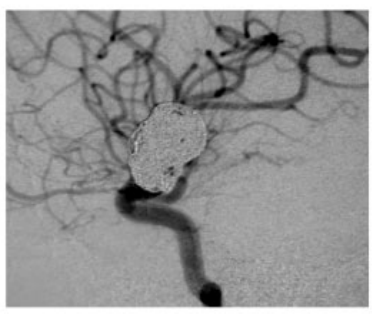

(h)

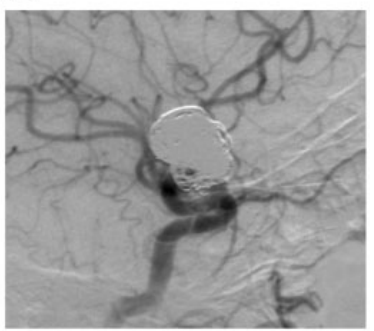

(I)

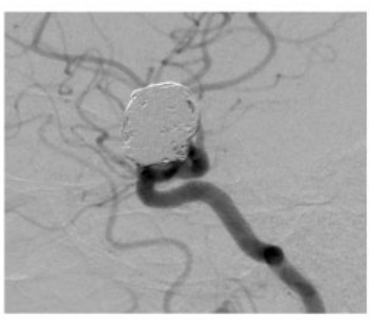

(m)

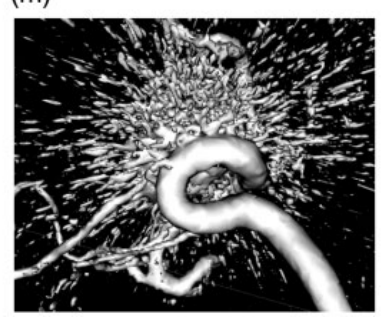

Figure 1. Recanalization after coiling: A patient with a ruptured right internal carotid artery (ICA) ophthalmic segment aneurysm: Axial CT slice (a) showed the aneurysm and frontal hematoma adjacent to the aneurysm. A large ICA ophthalmic segment aneurysm projecting cranially was seen on lateral $2 \mathrm{D}$ (b) and $3 \mathrm{D}$ (c) angiograms. There was also a small aneurysm bleb (c, arrow) just before the ophthalmic artery on the medial wall. The aneurysm was treated with coil embolization, and a small remnant was left in the neck (d). Six-monthfollow-up angiograms revealed significant recanalization in the ruptured one (e) and some growth of the small aneurysm (f, arrow). The recanalized part and the small aneurysm were treated with a Silk flow diverter without coiling $(\mathrm{g})$. While angiograms performed 6 (h) and 12 (i) months later showed a small aneurysm filling at the neck, angiograms after 18 (j) and 66 (k, I) months showed complete closure of the large and small aneurysms $(\mathrm{m})$. 


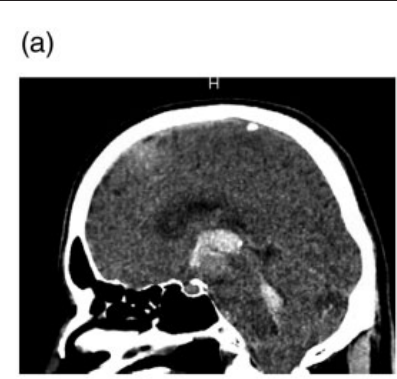

(e)

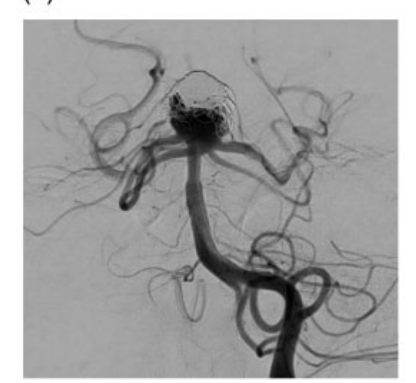

(i)

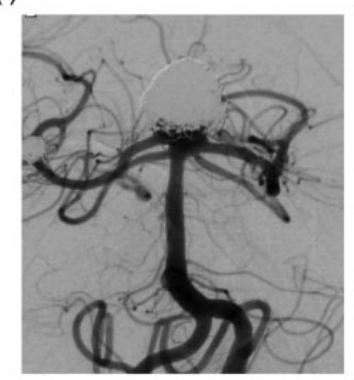

(m)

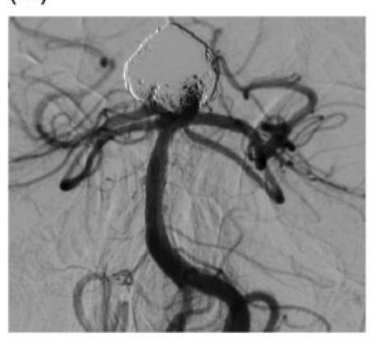

(b)

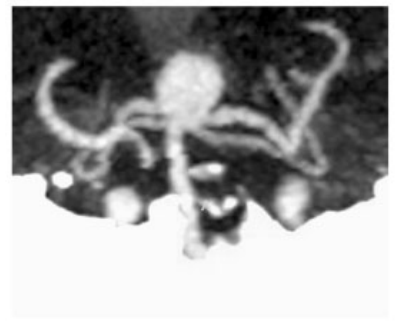

(f)

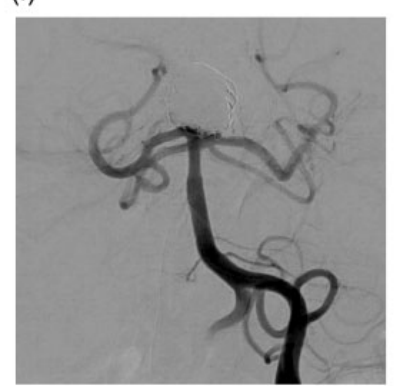

(j)

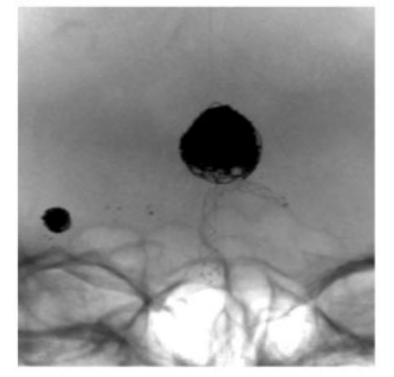

(n)

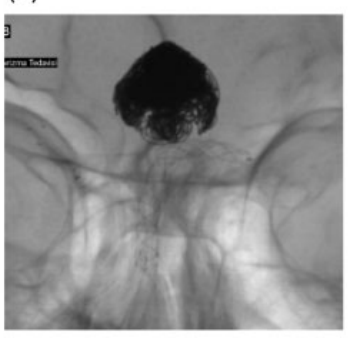

(c)

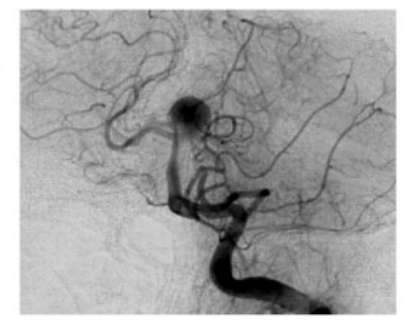

(g)

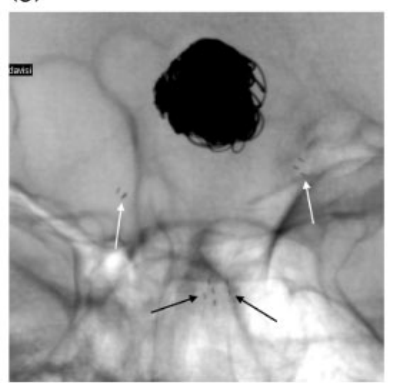

(k)

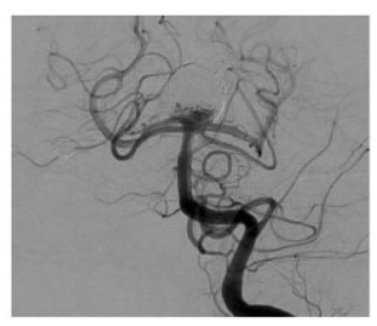

(d)

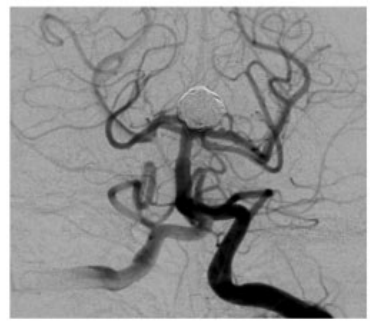

(h)

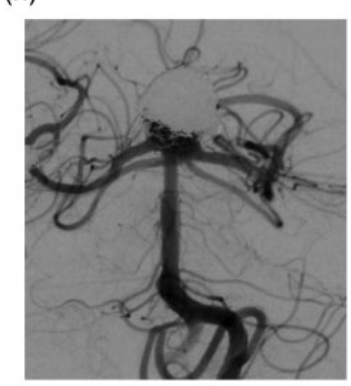

(I)

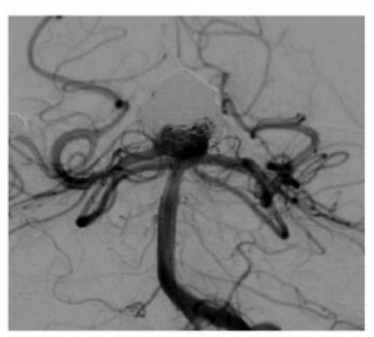

(o)

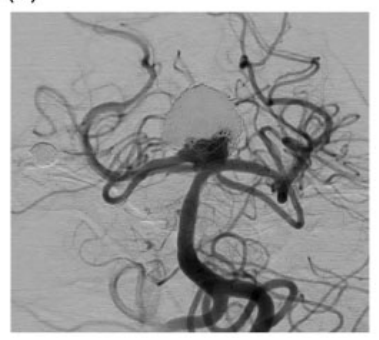

Figure 2. Recanalization after stent-assisted coiling: A patient with a ruptured basilar tip aneurysm: A sagittal CT section (a), CT angiography (b), and DSA (c) showed subarachnoid hemorrhage next to the aneurysm and inside the ventricles (a), and wide-necked saccular aneurysm that bilateral posterior cerebral and superior cerebellar arteries came out from the aneurysm neck (b). The aneurysm was completely coiled with a remodeling balloon (d). A large recurrence at the aneurysm neck six months later was seen (e) and treated with Y-stent-assisted coiling (f) using two Neuroform Atlas stents (Stryker). Arrows in (g) denote the proximal (black) and distal (white) markers of the stents. In the same session, the patient's small, right middle cerebral artery bifurcation aneurysm was also treated with stent-assisted coiling using the same stent, Neuroform Atlas (not shown here). Follow-up DSAs performed 6 and 12 (h) months later showed a recanalization increasing. This recanalization was treated with stent-assisted coiling using two telescoped Leo stents (Balt) extending from the basilar artery to the left posterior cerebral artery (i, j). Recanalization and growth of the aneurysm were seen again on 6- and 18-month angiograms ( $\mathrm{k}, \mathrm{l})$. This time, the aneurysm was subtotally embolized $(\mathrm{m})$, and a Pipeline Shield flow diverter (Medtronic) was inserted from the basilar artery to the left posterior cerebral artery, inside the telescopically deployed Leo stents ( $n$ ). Remnant growth of the basilar tip (o) was seen on follow-up angiograms performed 6 months later.

of aneurysms results in a gradual yet more complete aneurysm obliteration, especially in large and giant aneurysms. As shown in many previous studies, ${ }^{17-19}$ it is expected that the branches originating from the area covered by the FDS remain open.
An inflammatory reaction occurs due to the flow stasis inside the aneurysm, then thrombosis and healing of the aneurysm are accomplished. ${ }^{17}$ The stent aids in neointimal proliferation and remodeling of the parent vessel simultaneously. In studies 
investigating the efficacy and safety of FDS, excellent results were obtained, and these studies led to increased utilization of FDSs in a wide range of aneurysm types. ${ }^{18,19}$ In addition to the intracranial complex, wide-necked large and giant, fusiform, blister and bifurcation aneurysms, ${ }^{8,10,12,20,21}$ another accepted FDS indication of the aneurysm treatment, the efficacy and safety of which were shown by some studies, is the recanalized and residual aneurysms previously treated with other techniques, coiling or stentassisted coiling. ${ }^{13,15,16,22-24}$ When complete aneurysm occlusion is obtained with FDSs, recanalization is rare. This feature is a valid reason for choosing them for recurrent or residual aneurysms ${ }^{25}$ because the recanalization of coiled or stent-assisted-coiled aneurysms is not negligible. About $20 \%$ of coiled aneurysms ${ }^{26}$ and $12 \%$ of stent-assisted-coiled ones recur, ${ }^{27}$ and half of all recurrences require retreatment. Rebleeding from the aneurysms treated endovascularly with coiling or stent-assisted coiling is low but if it occurs, it may result in catastrophe. A study by Munich et al. ${ }^{28}$ including 1292 aneurysms treated with primary coiling or stent-assisted coiling showed residual filling immediately after treatment in almost $50 \%$ (n: 626) of the cases. Of these aneurysms, 13 ruptured during the follow-up period (mean $7.3 \mathrm{mo}$ ) and 11 of them $(84.6 \%)$ were ruptured aneurysms. They concluded that while unruptured aneurysms with a remnant after endovascular treatment had a very low risk of rupture $(0.6 \%)$, ruptured aneurysms had a higher risk of rerupture (3.4\%) from a neck remnant. In their systematic review and metaanalysis, given a $5 \%$ retreatment rate, Rizvi et al. ${ }^{29}$ showed that spontaneous rupture of previously unruptured, small- and medium-sized, well-treated aneurysms was exceedingly rare $(0.25 \%)$ after coiling. However, it should be taken into consideration that the retreatment of aneurysms after endovascular treatment due to the growing residual or recanalization prevents to be faced more reruptured cases. Therefore, in such conditions, retreatment of those aneurysms should be done, as in our cohort including the growing remnant and recanalized aneurysms that had mostly ruptured at presentation $(74.7 \%$ of all cases). In a pooled analysis of three large studies by Kallmes et al., ${ }^{30}$ endovascular treatment of intracranial aneurysms with the PED was safe and effective with a combined major morbidity and neurological mortality rate of $7.1 \%$. Because rerupture of the residual aneurysms is quite low when we compare the complication rate of endovascular treatment with FDSs, stable residual and recanalized endovascularly treated aneurysms can be followed under close attention. Beyond the serious life-threatening intracranial bleeding from aneurysms or brain parenchyma, or internal bleeding from other conditions such as epistaxis, melena, hematuria; minor bleeding or "nuisance bleeding" (that is, petechia, ecchymosis, and bleeding from small cuts or bruises) may require reducing the dose or cessation of the antiplatelet therapy. ${ }^{31}$ Several studies related to cardiac interventions requiring antiplatelet therapy have been published in recent years, ${ }^{32,33}$ and a study by Pressman et al. ${ }^{31}$ revealed that nuisance bleeding events occurred in one-third of the patients with intracranial aneurysms treated with PED on dual antiplatelet therapy; moreover, this was more common at older ages. So, nuisance bleeding after treatment with FDSs should be taken into consideration as well, especially in older patients, when endovascular treatment with FDSs will be decided.

How we treat recanalized aneurysms and those ruptured aneurysms that were suboptimally coiled in the acute phase to prevent rebleeding, without targeting an initial complete occlusion, remains a problem. ${ }^{13}$ Retreatment of recanalized or residual aneurysms treated endovascularly can be performed either microsurgically or endovascularly. However, there has been a tendency for the endovascular retreatment of these aneurysms due to surgical difficulties created by the previous endovascular procedure (presence of embolic materials, such as coils or stents). The presence of comorbidities in addition to the recurrent aneurysm and the presence of a recanalized posterior circulation aneurysm are additional reasons for preferring endovascular treatment. Microsurgery may be reserved for complex recanalized aneurysms that need an external-internal carotid bypass, in patients with uncorrectable coagulation disorders, or if antiplatelet agents are contraindicated. ${ }^{34}$ Waldron et al. ${ }^{35}$ treated 43 aneurysms with recanalized or residual aneurysms. Of these, 33 were managed with clipping, 7 were bypassed, and 3 were wrapped. While permanent morbidity was $2 \%$, surgical mortality was $7 \%$. However, with endovascular retreatment, morbimortality is very low in larger series. ${ }^{11,13,14,20,23}$ In our study, which encompassed recanalized and residual aneurysms after endovascular treatment with FDSs, the overall aneurysm obliteration rate was $87.4 \%$. Of the 11 incompletely occluded aneurysms, 3 were the bifurcation aneurysms. A side branch coming from the neck of the aneurysm might hinder the complete closure of the aneurysms. This is because the occlusion of the aneurysm depends on whether the territory supplied by this branch receives a collateral supply or not, as has been stated in some studies. ${ }^{12,36}$ We noted a technical problem in one $(1.2 \%)$ patient and stent thrombosis in two $(2.2 \%)$ patients, but despite in-stent stenosis requiring balloon angioplasty at one year of follow-up, permanent neurological complications and mortality were zero. Table 3 shows a summary of the studies related to flow diversion in recanalized or residual aneurysms.

There have been a few studies focusing on the endovascular treatment of recanalized or residual aneurysms after coiling or stent-assisted coiling. In one such work, Daou et al. ${ }^{13}$ treated 33 recanalized 
Table 3. Studies showing flow diverter treatment of residual or recanalized aneurysms treated endovascularly.

\begin{tabular}{|c|c|c|c|c|c|c|c|c|c|c|}
\hline \multirow[b]{2}{*}{ Study } & \multirow{2}{*}{$\begin{array}{l}\mathrm{N} \text { of } \\
\text { aneu. }(\mathrm{P})\end{array}$} & \multicolumn{3}{|c|}{ Previous treatment } & \multirow[b]{2}{*}{$\mathrm{FD}$} & \multirow{2}{*}{$\begin{array}{l}\text { Follow-up } \\
\text { period }(\mathrm{m})\end{array}$} & \multirow{2}{*}{$\begin{array}{l}\text { Complete } \\
\text { closure rate }(\%)\end{array}$} & \multicolumn{3}{|c|}{ Complications (\%) } \\
\hline & & Coil & Stent + coil & Surg. & & & & Tech. & Morb. & Mort. \\
\hline Benaissa et al. ${ }^{12}$ & $29(29)$ & 23 & 6 & - & $\mathrm{S}, \mathrm{P}$ & $5(3-21)$ & 60.7 & 12.7 & 17.2 & 0 \\
\hline Dau et al. ${ }^{11}$ & $33(33)$ & 33 & - & - & $P$ & $8(6-16)$ & 76.7 & 0 & 3 & 0 \\
\hline Heiferman et al. ${ }^{22}$ & $25(25)$ & - & 25 & - & $P$ & 12 & 38 & 12 & 4 & 0 \\
\hline Kühn et al. ${ }^{14}$ & $24(24)$ & 18 & - & 6 & $P$ & 6 & 66.7 & 4.2 & 0 & 0 \\
\hline Dau et al. ${ }^{21}$ & $21(21)$ & - & 21 & - & $P$ & 10.4 & 55.6 & 0 & 9.5 & 4.8 \\
\hline Dornbos et al. ${ }^{26}$ & $13(13)$ & 7 & 2 (FD) & 4 & $\mathrm{P}$ & $26.1(6-53)$ & 100 & 0 & 0 & 0 \\
\hline Zhang et al. ${ }^{24}$ & $8(8)$ & 1 & 7 & - & $\mathrm{P}$ & $16.9(7-36)$ & 71.4 & 0 & 0 & 0 \\
\hline Bender et al. ${ }^{20}$ & $20(18)$ & - & 20 (13 FD) & - & $P$ & $13.1(5-51)$ & 56.0 & 0 & 5 & 5 \\
\hline Park et al. ${ }^{23}$ & $17(17)$ & - & 17 & - & $\mathrm{P}$ & $22(6-48)$ & 94.1 & 0 & 0 & 0 \\
\hline Current study & $87(86)$ & 69 & 18 & - & $\mathrm{S}, \mathrm{D}, \mathrm{SP}, \mathrm{F}, \mathrm{P}$ & $21.0(2-96)$ & 87.4 & 1.2 & 0 & 0 \\
\hline
\end{tabular}

N: Number; aneu: Aneurysm; P: Patient; Surg: Surgery; m: Month, Tech: Technical; Morb: Morbidity; Mort: Mortality; S: Silk; P: pipeline; D: Derivo; SP: Surpass, F: FRED.

aneurysms after coiling. Their result was excellent, with a $76.7 \%$ complete and a 10\% near-complete occlusion rate. Of these aneurysms, 53\% were ruptured. They did not encounter any technical or neurological complications. In our series, which had a similar rate of coiled patients $(79.3 \%)$, the angiographic cure rate was $87.4 \%$, which is higher than that reported by Daou et al. ${ }^{13}$ In fact, the rate of previously coiled ruptured aneurysms was similar to that found by other studies on the retreatment of a recanalized aneurysm. ${ }^{14,16,37}$ This is to be expected, as endovascular treatment of a ruptured aneurysm with only coiling or balloon-assisted coiling may be regarded as rescue therapy. The main purpose of treating ruptured aneurysms in the acute phase is to prevent rebleeding. Therefore, aggressive coiling may not be warranted in an effort to decrease complications, reserving complete treatment for the chronic phase, in which there is a very low or no risk of rebleeding. Another reason for the increased rate of recanalized or residual aneurysms after coiling is the high rate of aneurysm recanalization (up to $50 \%$ ) associated with coiling only. ${ }^{13,16,19}$

Some studies evaluated flow diversion treatment of recanalized, or remnant aneurysms seen after stentassisted coiling. According to the results of certain works, ${ }^{19,22-24}$ a stent seems to negatively affect the results, with occlusion rates from $38 \%$ to $65 \%$ and morbidity ranging from 0 to $14.3 \%$. The stent increases technical problems, such as in-stent navigation and FD deployment. Incomplete FD opening caused by a previous stent results in additional complications. Additionally, the flow diversion effect may be reduced by the stent. In studies by Heiferman et al., ${ }^{24}$ Daou et al., ${ }^{23}$ Zhang et al., ${ }^{34}$ Bender et al., ${ }^{22}$ and Park et al. ${ }^{25}$ post-stent recanalized or residual aneurysms were treated with FDSs. Although Heiferman et al., ${ }^{24}$ Daou et al., ${ }^{23}$ and Bender et al. ${ }^{22}$ suggested a low obliteration rate $(38 \%, 55.6 \%$, and $56 \%$, respectively) of post-stent recanalized aneurysms with flow diversion, in the studies by Zhang et al. ${ }^{34}$ and Park et al., ${ }^{25}$ the closure rate was fairly high, at $71.4 \%$ and $94.1 \%$, respectively. In the current study, the closure rate of the subgroup of 18 previously stented aneurysms was $77.8 \%$, better than those of previous works, with the exception of Park et al. Our study is unique in two aspects. First of all, none of the previous authors compared the results of FDSs in patients with different initial treatment modalities. Our statistical analysis showed no significant difference between subgroups treated with coiling and stent-assisted coiling. However, the rate of residuals after FDS placement for post-stent cases was numerically higher. Therefore, it is reasonable to state that either the presence of a previous stent does not affect the results of FDSs or the effect of the stent, if actually present, was not shown because the size of our cohort to date is not yet large enough to show a small difference. In either case, the decision to proceed with FDSs in post-stent aneurysms is not altered, given the lack of efficacy of other modalities. On the other hand, it should be noted that both in our series (treated with nine braided and nine laser-cut stents) and that of Park et al., who reported a higher rate of complete occlusion after stenting, not only laser-cut but also braided stents were used (six braided and six lasercut, stents) in contrast to the other papers mentioned above. Braided stents are well-known for their ability to appose the wall and may well be the underlying reason for the better results after retreatment with FDSs. The use of laser-cut stents in three of the four recanalized aneurysms treated with stent-assisted coiling supports this finding. The second difference is that the earlier reports included only the first generation of stents (Silk and Pipeline) only. Neurointerventional devices have improved rapidly in recent years, including new generation, less thrombogenic, easily deployable FDSs, distal access guiding and intermediate catheters, and highly sophisticated microcatheters. ${ }^{25}$ This may also be a reason for the superior results obtained in our study, in which a wide range of devices was used (Table 3). Despite the remarkable size of our 
cohort, more research is still needed to be able to prove this assumption due to the limited number of post-stent residual/recurrent aneurysms treated by FDSs reported to date. ${ }^{22,25}$

In the literature, the morbidity and mortality of aneurysm treatment with FDSs vary from $3 \%$ to $22.4 \%$, and from $0 \%$ to $8 \%$, respectively. ${ }^{17,30}$ However, in the PREMIER study, Hanel et al. ${ }^{7}$ showed that if the aneurysm size was small- or medium-sized (less or equal to $12 \mathrm{~mm}$ ), the morbimortality was very low $(2.4 \%)$. In our series, beyond a technical complication, we encountered two stent thromboses that were treated during the procedure without causing any neurological problems. Additionally, in one patient, in-stent stenosis necessitated balloon angioplasty 1 year after treatment. This did not lead to any neurological deterioration. Overall morbimortality was zero as in similar studies with FDS treatment of recanalized and residual aneurysms performed by Kühn et al., ${ }^{16}$ Dornbos et al., ${ }^{37}$ Zhang et al., ${ }^{34}$ and Park et al. ${ }^{25}$ However, in studies by Benaissa et al., ${ }^{14}$ Daou et al., ${ }^{23}$ and Bender et al., ${ }^{22}$ while morbidity was between $3.0 \%$ and $17.2 \%$, mortality was approximately $5 \%$ due to intracerebral hemorrhage and brain stem infarction that occurred in two patients in two separate studies. Although a direct comparison of complications in retreatments using FDS with aneurysms primarily treated by flow diversion is not appropriate, we can expect a lower rate of permanent neurological complications and mortality occurrence in retreatments. This may be because it is perhaps easier to deploy FDS in a previously placed stent scaffold, and no additional manipulation to fill the aneurysm with coils is required in most cases. Additionally, residual/recurrent aneurysms tend to be small and, generally, the vulnerable part of the dome of the aneurysms has been filled with coils. Therefore, no late aneurysmal hemorrhage is expected secondary to the aneurysm thrombosis. Although our study included large and giant aneurysms, the mean initial size of the aneurysms treated coiling or stent-assisted coiling was $10.3 \mathrm{~mm}(3-40 \mathrm{~mm})$. We can assume that the residual/recanalized aneurysms were significantly smaller than the initial size. This assumption can explain the low rate of morbimortality in our cases, as found in the PREMIER study that showed lower morbimortality in small- or medium-sized aneurysms treated with FDSs than other FDS studies including large and giant aneurysms. ${ }^{7,30}$

\section{Study limitations}

The study was retrospective and there was no control group. Aneurysms locations and FDSs used in the treatment were not standardized.

\section{Conclusion}

FDSs are safe, effective, and feasible in the treatment of recanalized or residual intracranial aneurysms treated endovascularly. A low rate of complications makes flow diversion a preferred first-line treatment method in those aneurysms.

\section{Authors' contribution}

All authors aided in the collection of data. EA, BH, KA and AA wrote the article.

\section{Ethical approval}

Istanbul Medipol University Clinical Research Institutional Ethics Committee approval number: 10840098-604.01.01.E9268

\section{Declaration of conflicting interests}

The author(s) declared no potential conflicts of interest with respect to the research, authorship, and/or publication of this article.

\section{Funding}

The author(s) received no financial support for the research, authorship, and/or publication of this article.

\section{ORCID iD}

Erol Akgul (D) https://orcid.org/0000-0003-0020-3759

\section{References}

1. Nelson PK, Lylyk P, Szikora I, et al. The pipeline embolization device for the intracranial treatment of aneurysms trial. AJNR Am J Neuroradiol 2011; 32: 34-40.

2. Cirillo L, Leonardi M, Dall M, et al. Complications in the treatment of intracranial aneurysms with silk stents: an analysis of 30 consecutive patients. Interv Neuroradiol 2012; 18: 413-425.

3. Kallmes DF, Hanel R, Lopes D, et al. International retrospective study of the pipeline embolization device: a multicenter aneurysm treatment study. $\mathrm{Am} \mathrm{J}$ Neuroradiol 2015; 36: 108-115.

4. Becske T, Brinjikji W, Potts MB, et al. Long-term clinical and angiographic outcomes following pipeline embolization device treatment of complex internal carotid artery aneurysms: five-year results of the pipeline for uncoilable or failed aneurysms trial. Neurosurgery 2017; 80: 40-48.

5. Briganti F, Leone G, Cirillo L, et al. Postprocedural, midterm, and long-term results of cerebral aneurysms treated with flow-diverter devices: 7-year experience at a single center. Neurosurg Focus 2017; 42: 1-8.

6. Cagnazzo F, Mantilla D, Rouchaud A, et al. Endovascular treatment of very large and giant intracranial aneurysms: comparison between reconstructive and deconstructive techniques - a meta-analysis. AJNR Am J Neuroradiol 2018; 39: 852-858.

7. Hanel RA, Kallmes DF, Lopes DK, et al. Prospective study on embolization of intracranial aneurysms with the pipeline device: the PREMIER study 1 year results. J NeuroIntervent Surg 2020; 12: 62-66. 
8. Chalouhi N, Starke RM, Yang S, et al. Extending the indications of flow diversion to small, unruptured, saccular aneurysms of the anterior circulation. Stroke 2014; 45: 54-59.

9. Burkhardt JK, Benet A and Lawton MT. Management of small incidental intracranial aneurysms. Neurosurg Clin N Am 2017; 28: 389-396.

10. Brouillard AM, Sun X, Siddiqui AH, et al. The use of flow diversion for the treatment of intracranial aneurysms: expansion of indications. Cureus 2016; 8: 1-8.

11. Peschillo S, Cannizzaro D, Caporlingua A, et al. A systematic review and Meta-analysis of treatment and outcome of blister-like aneurysms. AJNR Am J Neuroradiol 2016; 37: 856-861.

12. Topcuoglu OM, Akgul E, Daglioglu E, et al. Flow diversion in middle cerebral artery aneurysms: is it really an all-purpose treatment? World Neurosurg 2016; 87: 317-327.

13. Daou B, Starke RM, Chalouhi N, et al. The use of the pipeline embolization device in the management of recurrent previously coiled cerebral aneurysms. Neurosurgery 2015; 77: 692-697.

14. Benaissa A, Januel AC, Herbreteau D, et al. Endovascular treatment with flow diverters of recanalized and multitreated aneurysms initially treated by endovascular approach. J Neurointerv Surg 2015; 7: 44-49.

15. Mai JC and Hoh BL. Endovascular management of recurrent aneurysms. Neurol Res 2014; 36: 323-331.

16. Kühn AL, De Macedo Rodrigues K, Lozano JD, et al. Use of the pipeline embolization device for recurrent and residual cerebral aneurysms: a safety and efficacy analysis with short-term follow-up. J Neurointerv Surg 2017; 9: 1208-1213.

17. Akgul E, Onan HB, Akpinar S, et al. The DERIVO embolization device in the treatment of intracranial aneurysms: short- and midterm results. World Neurosurg 2016; 95: 229-240.

18. Park W, Song Y, Park KJ, et al. Hemodynamic characteristics regarding recanalization of completely coiled aneurysms: Computational fluid dynamic analysis using virtual models comparison. Neurointervention 2016; 11: 30-36.

19. Limbucci N, Leone G, Renieri L, et al. Expanding indications for flow diverters: distal aneurysms, bifurcation aneurysms, small aneurysms, previously coiled aneurysms and clipped aneurysms, and carotid cavernous fistulas. Neurosurgery 2020; 86: S85-94.

20. Meling TR. What are the treatment options for blisterlike aneurysms? Neurosurg Rev 2017; 40: 587-593.

21. Strickland BA, Rennert RC, Bakhsheshian J, et al. Extracranial-intracranial bypass for treatment of blister aneurysms: efficacy and analysis of complications compared with alternative treatment strategies. World Neurosurg 2018; 117: e417-24-e424.

22. Bender MT, Vo CD, Jiang B, et al. Pipeline embolization for salvage treatment of previously stented residual and recurrent cerebral aneurysms. Interv Neurol 2018; 7: 359-369.

23. Daou B, Starke RM, Chalouhi N, et al. Pipeline embolization device in the treatment of recurrent previously stented cerebral aneurysms. AJNR Am J Neuroradiol 2016; 37: 849-855.

24. Heiferman DM, Billingsley JT, Kasliwal MK, et al. Use of flow-diverting stents as salvage treatment following failed stent-assisted embolization of intracranial aneurysms. J Neurointerv Surg 2016; 8: 692-695.

25. Park KY, Yeon JY, Kim BM, et al. Efficacy and safety of flow-diverter therapy for recurrent aneurysms after stent-assisted coiling. AJNR Am J Neuroradiol 2020; 41: 663-668.

26. Ferns SP, Sprengers MES, Van Rooij WJ, et al. Coiling of intracranial aneurysms: a systematic review on initial occlusion and reopening and retreatment rates. Stroke 2009; 40: e523-529-e529.

27. Chalouhi N, Jabbour P, Singhal S, et al. Stent-assisted coiling of intracranial aneurysms: predictors of complications, recanalization, and outcome in 508 cases. Stroke 2013; 44: 1348-1353.

28. Munich SA, Cress MC, Rangel-Castilla L, et al. Neck remnants and the risk of aneurysm rupture after endovascular treatment with coiling or stent-assisted coiling: much ado about nothing? Clin Neurosurg 2019; 84: 421-427.

29. Rizvi A, Seyedsaadat SM, Alzuabi M, et al. Long-term rupture risk in patients with unruptured intracranial aneurysms treated with endovascular therapy: a systematic review and Meta-analysis. AJNR Am J Neuroradiol 2020; 41: 1043-1048.

30. Kallmes DF, Brinjikji W, Cekirge S, et al. Safety and efficacy of the pipeline embolization device for treatment of intracranial aneurysms: a pooled analysis of 3 large studies. J Neurosurg 2017; 127: 775-780.

31. Pressman E, De la Garza CA, Chin F, et al. Nuisance bleeding complications in patients with cerebral aneurysm treated with Pipeline embolization device. Journal of NeuroInterventional Surgery. Published Online First: 3 July 2020. https://doi.org/10.1136/neurintsurg-2020016245.

32. Ben-Dor I, Torguson R, Scheinowitz M, et al. Incidence, correlates, and clinical impact of nuisance bleeding after antiplatelet therapy for patients with drug-eluting stents. Am Heart J 2010; 159: 871-875.

33. Armero S, Bonello L, Berbis J, et al. Rate of nuisance bleedings and impact on compliance to prasugrel in acute coronary syndromes. Am J Cardiol 2011; 108: 1710-1713.

34. Zhang Y, Huang QH, Fang Y, et al. A novel flow diverter (tubridge) for the treatment of recurrent aneurysms: a single-center experience. Korean J Radiol 2017; 18: 852-859.

35. Waldron JS, Halbach VV and Lawton MT. Microsurgical management of incompletely coiled and recurrent aneurysms: trends, techniques, and observations on coil extrusion. Oper Neurosurg 2009; 64: 301-315.

36. Bhogal P, AlMatter M, Bäzner H, et al. Flow diversion for the treatment of MCA bifurcation aneurysms - a single centre experience. Front Neurol 2017; 8: 20-28.

37. Dornbos D, Karras CL, Wenger N, et al. Pipeline embolization device for recurrence of previously treated aneurysms. Neurosurg Focus 2017; 42: 1-7. 